\title{
Integrated analysis of pain, health-related quality of life, and analgesic use in patients with metastatic castration-resistant prostate cancer treated with Radium-223
}

\author{
Sushil K. Badrising $D^{1,14}$, Rebecca D. Louhanepessy ${ }^{1,14}$, Vincent van der Noort ${ }^{2}$, Jacobien Kieffer ${ }^{3}$, Jules L. L. M. Coenen ${ }^{4}$, \\ Paul Hamberg ${ }^{5}$, Aart Beeker ${ }^{6}$, Nils Wagenaar ${ }^{7}$, Marnix Lam $^{8}$, Filiz Celik (D) $^{9}$, Olaf J. L. Loosveld ${ }^{10}$, Ad Oostdijk ${ }^{11}$, Hanneke Zuetenhorst ${ }^{5}$, \\ Jeantine M. de Feijter ${ }^{1}$, Vincent O. Dezentjé ${ }^{1}$, Suzan Ras-van Spijk ${ }^{5}$, Erik Vegt ${ }^{12}$, John B. Haanen ${ }^{1}$, Lonneke V. van de Poll-Franse ${ }^{3}$, \\ Wilbert Zwart ${ }^{13 凶}$ and Andries M. Bergman (iD ${ }^{1,13 凶}$
}

(c) The Author(s) 2021

BACKGROUND: Radium-223 (Ra-223), an alpha-emitting radiopharmaceutical, established an improved overall survival and healthrelated quality of life (HRQoL) in symptomatic metastatic castration-resistant prostate cancer (mCRPC) patients. However, effects on pain were not specifically evaluated. Here we assess integrated HRQoL, pain, and opioid use in a contemporary, more extensively pretreated, symptomatic and asymptomatic MCRPC population.

METHODS: mCRPC patients scheduled for Ra-223 treatment were included and analyzed for HRQoL, pain, and opioid use, using Functional Assessment of Cancer Therapy-Prostate (FACT-P) and Brief Pain Inventory-Short Form (BPI-SF) questionnaires and recording of opioid use and dosage, respectively. Primary outcome measure was the percentage of patients experiencing a complete pain response (score of 0 on the BPI-SF Worst pain item and no increase in daily use of analgesics). A complete or partial pain response (better BPI-SF score and decrease in opioid use) and a better or no change in HRQoL was evaluated as an integrated overall clinical response (IOCR). Secondary endpoints included the time to pain progression (TPP) and Total FACT-P deterioration (TTFD), defined as time from first Ra-223 treatment to clinical meaningful increase in BPI-SF Worst pain item score and Total FACT-P score, respectively.

RESULTS: This registry included 300 patients, of whom 105 (35\%) were evaluable for FACT-P and BPI-SF during Ra-223 treatment. Forty-five (43\%) patients had pain at baseline (PAB) (BPI-SF Worst pain score 5-10 points) and 60 (57\%) had no pain at baseline (noPAB) (BPI-SF Worst pain score $0-4$ points). Complete pain response was achieved in $31.4 \%$ of the patients, while $58 \%$ had an IOCR. The median TTP and TTFD were 5.6 and 5.7 months, respectively, while the difference between PAB and no-PAB patients was not significant.

CONCLUSIONS: In contemporary, extensively pretreated mCRPC patients, Ra-223 treatment induced complete pain responses while integrated analysis of HRQoL, pain response, and opioid use demonstrated that the majority of patients derive clinical benefit.

Prostate Cancer and Prostatic Diseases (2022) 25:248-255; https://doi.org/10.1038/s41391-021-00412-6

\section{INTRODUCTION}

Each year, over 1.2 million men are diagnosed with prostate cancer worldwide and approximately 350,000 patients succumb to the consequences of this disease, rendering it the most common noncutaneous cancer in males and the second largest cause of cancerrelated death in men [1]. Metastatic castration-resistant prostate cancer ( $\mathrm{mCRPC}$ ) is the end stage of this disease with high morbidity and mortality as hallmarks [2]. Up to $90 \%$ of mCRPC patients develop bone metastases, which are not only associated with a shorter life expectancy, but also with cancer-related pain and skeletal-related events, including pathological fractures, compression of the spinal cord, vertebral instability, and hypercalcemia, which all affect health-related quality of life (HRQoL) [3]. Symptoms and complications of bone metastases can be treated with analgesics, external beam-radiation therapy (EBRT), bisphosphonates, RANK-ligand inhibitors, surgery, and radiopharmaceuticals [4].

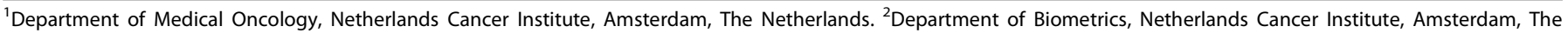

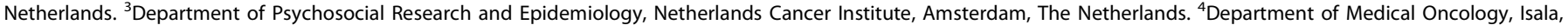

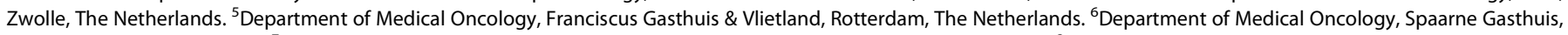

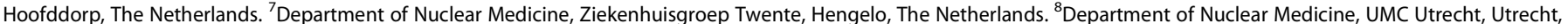

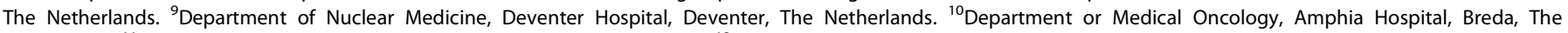

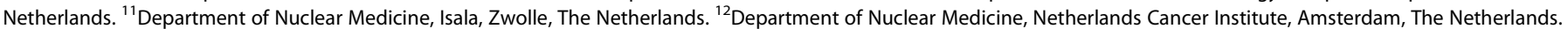
${ }^{13}$ Division of Oncogenomics, Netherlands Cancer Institute, Amsterdam, The Netherlands. ${ }^{14}$ These authors contributed equally: Sushil K. Badrising, Rebecca D. Louhanepessy. 凶email: w.zwart@nki.nl; a.bergman@nki.nl
} 
In the ALSYMPCA study, the alpha-emitter Radium-223 dichloride (Ra-223) showed a 3.6 month overall survival (OS) benefit and favorable HRQoL in symptomatic MCRPC patients [4]. However, the effect of Ra-223 on pain was not evaluated using pain-specific questionnaires, and changes in the dosages of analgesics were not considered in the evaluation of pain [5]. Another study showed that asymptomatic MCRPC patients treated with Ra-223 had better treatment outcomes than symptomatic patients, but HRQoL and pain were not assessed [6]. Since completion of the accrual of patients into the ALSYMPCA study, the number of treatment options for mCRPC patients has expanded significantly. Consequently, contemporary patients treated with Ra-223 are more extensively pretreated with novel agents, like abiraterone, enzalutamide, and cabazitaxel [7]. This questions the relevance of HRQoL results from the ALSYMPCA for present $\mathrm{mCRPC}$ patients [8]. Given the paucity of knowledge of the effect of Ra-223 on pain and $\mathrm{HRQoL}$ in contemporary symptomatic and asymptomatic $\mathrm{mCRPC}$ patients, there is a need for a re-evaluation [8].

The primary objective of this prospective observational study was to evaluate the efficacy of Ra-223 on patient-reported pain and analgesics use. Secondly, we performed an integrated analysis of the effect of Ra-223 on patient-reported pain, analgesic use, and HRQoL in a contemporary real-life cohort. Since in daily practice, both patients with pain at baseline (PAB) and no-pain at baseline (no-PAB) are treated with Ra-223, these subgroups were assessed separately [9].

\section{METHODS}

\section{Study population and design}

A non-interventional, multicenter, prospective observational registry was initiated to evaluate clinical outcomes, HRQoL, pain, and analgesic use in a real-life MCRPC population treated with Ra-223. The study design is fully described elsewhere [8]. In short, patients with progressive MCRPC and scheduled for Ra-223 treatment were included prospectively in 20 hospitals in the Netherlands (intention-to-treat population). There were no other inclusion and exclusion criteria or stopping rules. Paper questionnaires were sent to the patients 1 week before each treatment and in monthly follow-up, which were returned by mail to the data management office. This registry was approved by local medical ethics committees. Obtaining signed informed consent for the study was not required, but patients had to provide oral consent and written approval for registration and use of their identifiers.

\section{Procedures}

Patients were treated with Ra-223 at 4-week intervals. Dosing was according to the manufacturers' guidelines, which was a dose of $50 \mathrm{kBq}$ per kilogram of body-weight intravenously and from April 2016 on, $55 \mathrm{kBq}$ per kilogram of body-weight intravenously. Number of treatments was at the physician's discretion, who provided the motivation for discontinuation. Patients were evaluated at the outpatient clinic prior to each treatment, where performance scores and clinical lab assessments were documented. Radiological evaluation during and after Ra-223 treatment and frequency of follow-up visits were at the physician's discretion. Patients' baseline characteristics within 14 days prior to the first Ra-223 treatment were recorded. Baseline characteristics, efficacy assessments, and patient-reported outcome measures (PROMs) were stored in an electronic case-report form. Follow-up was continued until start of subsequent treatment or death. Data collection was performed on-site at the end of follow-up.

\section{Patient-reported outcome measures}

$\mathrm{HRQoL}$ and pain were assessed using the validated PROMs, Functional Assessment of Cancer Therapy-Prostate (FACT-P) and Brief Pain InventoryShort Form (BPI-SF), respectively [10-12]. Furthermore, patients were asked to list all analgesic drugs (free text: name, dose, frequency, and period of use) used in the previous 4 weeks. Patients were requested to complete all questionnaires at baseline and every 4 weeks during and after Ra-223 treatment until start of subsequent treatment or death. Patients were considered evaluable for pain, opioid use, and HRQoL analysis when baseline questionnaires and at least one set of questionnaires during treatment were returned. According to published algorithms, scale scores were calculated when at least $50 \%$ of the items in that scale had been completed [10-12]. An overview of the questionnaires and their use and interpretation is provided in Supplementary Table 1.

BPI-SF. The BPI-SF contains 4 items on pain severity (Worst pain, Least pain, Average pain, and Current pain) and 7 items on pain interference (e.g., during sleep, walking, daily activities) [10]. Every question is scored from 0 to 10 , where 0 is no pain/interference and 10 is the worst imaginable pain/interference (Supplementary Table 1). The clinically meaningful change of BPI-SF score (CMC-BPI) was defined as a change of score of at least $30 \%$ from baseline, with a minimum of 2 points $[10,11]$. Two groups in the cohort were separately analyzed; no-PAB patients were defined as a Worst pain score at baseline between 0 and 4 points, and PAB patients were defined as a Worst pain score between 5 and 10. This division is in line with the Initiative on Methods, Measurement and Pain Assessment in Clinical Trials (IMMPACT) recommendations [13].

FACT-P. The FACT-P is a validated 39-item questionnaire, including the FACT-General subscales: Physical well-being (PWB), Social/Family wellbeing (SWB), Emotional well-being (EWB), Functional well-being (FWB), and a prostate cancer subscale (PCS) [12]. Items are rated on a five-point scale ranging from 0 (not at all) to 4 (very much). Subscales as well as the total score can be calculated by the sum of the items. When not all subscales are evaluable, the total score cannot be calculated. The range of these scores is (0-156) for the FACT-P total score, $(0-28)$ for the PWB, SWB, and FWB, (0-24) for EWB, and (0-48) for PCS (Supplementary Table 1). The clinically meaningful change of FACT-P (CMC-FACT) was defined as a minimal change of 10 points from baseline for the Total FACT-P, 3 points from baseline for the subscales and 2 points from baseline for pain. A higher score indicates a better HRQoL [14].

Analgesic use. Patients were asked to fill out a list of all analgesics, dosages, and frequencies used in the past 4 weeks (Supplementary Table 1). Dosages of the various opioid drugs and formulations were converted to oral morphine equivalents in $\mathrm{mg}$ per day (Supplementary Table 2). Non-opioids and on-demand opioids were not included in our analysis.

\section{Endpoints and statistical analyses}

All endpoints were evaluated as changes in PROMs scores from baseline, meeting predefined criteria. The primary endpoint of the study was the percentage of patients experiencing a complete pain response. In line with the International Bone Metastases Consensus Working Party (IBMCWP), a complete pain response was defined as a score of 0 on the BPI-SF Worst pain item and no increase in daily use of analgesics; a partial response was defined as a pain reduction of at least 2 points on the BPI-SF Worst pain item or a reduction of at least $25 \%$ of daily use of analgesics; pain progression was defined as an increase in pain of at least 2 points on the BPI-SF Worst pain item or an increase of at least $25 \%$ of daily analgesic use. Indeterminate response was defined as all pain decreases, not captured by complete response or partial response [15]. Patients were categorized according to their best response.

Secondary endpoints included the percentage of patients experiencing a partial and an indeterminate pain response. Moreover, patients were categorized by their Total FACT-P response, which was "improved HRQoL" (better score meeting CMC-FACT), "no change in HRQoL" (no change or changes not meeting CMC-FACT), or "worse HRQoL" (deteriorated score meeting CMC-FACT). A complete or partial pain response and an improved $\mathrm{HRQOL}$ or no change in HRQoL were evaluated as an integrated overall clinical response (IOCR).

Moreover, secondary outcomes included time to Total FACT-P deterioration (TTFD), time to pain progression (TPP), progression-free survival (PFS), and OS. Definitions of the secondary endpoints are listed in Supplementary Table 3. All time-to-event endpoints were estimated using the KaplanMeier product limit method. Patients who did not experience an event of interest were censored at their last day of follow-up for OS and PFS and at the time of their last questionnaire for TTFD or TPP.

\section{Sample size calculation}

The rationale for sample size calculation is detailed in Supplementary Text 1. In short, a sample size of at least 120 evaluable patients was required to provide statistical power of $81 \%$ to detect significant increase in proportion of pain response rate compared to the placebo rate of $20 \%$. 
Table 1. Baseline characteristics of the registry sample and symptomatic and asymptomatic evaluable patients.

\begin{tabular}{|c|c|c|c|c|c|c|}
\hline \multirow[t]{2}{*}{ Patient demographics } & \multicolumn{6}{|c|}{ Median or value [IQR], no. of patients (\%) } \\
\hline & $\begin{array}{l}\text { Registry sample } \\
(n=300)\end{array}$ & $\begin{array}{l}\text { Evaluable sample } \\
(n=105)\end{array}$ & $p$ & $\begin{array}{l}\text { Pain at baseline } \\
(n=45)\end{array}$ & $\begin{array}{l}\text { No pain at baseline } \\
(n=60)\end{array}$ & $p$ \\
\hline Age, years & 73 [67-78] & 73 [68-77] & ns & 73 [68-77] & 72 [66-78] & ns \\
\hline $0-1$ & $264(88.0)$ & $94(90)$ & & $39(87)$ & $55(92)$ & \\
\hline 2 & $15(5.0)$ & $3(3)$ & & $2(4)$ & $1(2)$ & \\
\hline$\geq 3$ & 0 & 0 & & 0 & 0 & \\
\hline$\leq 7$ & $87(29.0)$ & $27(26)$ & & $10(22)$ & $17(28)$ & \\
\hline 8 & $67(22.3)$ & $32(30)$ & & $12(27)$ & $20(33)$ & \\
\hline$\geq 9$ & 95 (31.7) & $27(26)$ & & $14(31)$ & $13(22)$ & \\
\hline Missing data & $51(17.0)$ & $19(18)$ & & $9(20)$ & $10(17)$ & \\
\hline \multicolumn{7}{|l|}{ Metastatic sites, no. of patients (\%) } \\
\hline \multicolumn{3}{|c|}{ No. of bone metastases, no. of patients (\%) } & ns & & & ns \\
\hline $0-1$ & 0 & $2(2)$ & & $1(2)$ & $1(2)$ & \\
\hline $2-6$ & $21(7.0)$ & $12(11)$ & & $5(11)$ & $7(2)$ & \\
\hline$>6$ & $246(82.0)$ & $87(83)$ & & $37(82)$ & $50(80)$ & \\
\hline Super scan & $5(1.7)$ & $2(2)$ & & 0 & $2(3.1)$ & \\
\hline Missing data & $28(9.3)$ & $6(6)$ & & $3(7)$ & $3(5)$ & \\
\hline \multicolumn{7}{|l|}{ Laboratory values } \\
\hline PSA, $\mu \mathrm{g} / \mathrm{l}$ & $72.3[25.0-175.0]$ & 72 [22-179] & ns & 73 [16-225] & $72.0[23-172]$ & ns \\
\hline Hemoglobin, mmol/l & $12.6[11.3-13.4]$ & $12.6[11.6-13.4]$ & ns & $12.3[11.6-13.4]$ & $12.7[11.6-13.4]$ & ns \\
\hline ALP, U/I & $138[85-248]$ & 118 [75-242] & ns & $136[85-330]$ & $102[73-186]$ & ns \\
\hline $\mathrm{ALP} \geq 220 \mathrm{U} / \mathrm{l}, n(\%)$ & $81(27.0)$ & $28(27)$ & ns & $15(33)$ & $13(22)$ & ns \\
\hline 3 & $50(16.7)$ & $19(18)$ & & $4(9)$ & $15(25)$ & \\
\hline 4 & $13(4.3)$ & $5(5)$ & & $4(9)$ & $1(2)$ & \\
\hline 5 & $3(1.0)$ & $1(1)$ & & $1(2)$ & 0 & \\
\hline Missing data & 0 & $1(1)$ & & 0 & $1(2)$ & \\
\hline \multicolumn{7}{|c|}{ Specific previous treatments, no. of patients (\%) } \\
\hline Abiraterone and or Enzalutamide & $214(71.3)$ & $75(71)$ & ns & $31(69)$ & $44(73)$ & ns \\
\hline Docetaxel & $197(65.7)$ & $73(71)$ & ns & $35(78)$ & $38(63)$ & ns \\
\hline Cabazitaxel & $52(17.3)$ & $18(17)$ & ns & $10(22)$ & $8(13)$ & ns \\
\hline $\begin{array}{l}\text { Radiotherapy } 12 \text { weeks prior to } \\
\text { treatment }\end{array}$ & $23(8)$ & $2(2)$ & 0.01 & $2(4)$ & 0 & ns \\
\hline \multicolumn{7}{|c|}{ Concomitant medication, no. of patients (\%) } \\
\hline Bisphosphonates & 49 (16.7) & $11(10)$ & 0.03 & $3(7)$ & $8(13)$ & ns \\
\hline Denosumab & $63(24.4)$ & $25(24)$ & ns & $14(31)$ & $11(18)$ & ns \\
\hline Calcium/vitamin D & $123(41.0)$ & $55(52)$ & 0.02 & $25(56)$ & $30(50)$ & ns \\
\hline Analgesics use & & $n=103$ & & $n=44$ & $n=59$ & \\
\hline Non-opioids & NA & $3(2.9)$ & & $0(0)$ & $3(6.7)$ & \\
\hline Opioids & NA & $38(36)$ & & $25(56)$ & $13(22)$ & $<0.001$ \\
\hline Dose $(\mathrm{mg} / \text { day })^{\mathrm{a}}$ & NA & $44.4[18.8-111.6]$ & & 60 [15-118.8] & $30[30-75]$ & ns \\
\hline
\end{tabular}

Data are $n(\%)$, median or value [IQR]. ECOG Eastern Cooperative Oncology Group, PSA serum prostate-specific antigen, ALP serum alkaline phosphatase, $L D H$ lactate dehydrogenase, ns not significant, NA not available.

${ }^{\mathrm{a}}$ Oral morphine equivalent. 
Table 2. Patient-reported outcomes: median time to BPI-SF and FACT-P deterioration and pain response.

Outcome variables

Evaluable sample
$(n=105)$

Median [IQR], no. of patients (\%) [IQR or $95 \% \mathrm{CI}]$ $(n=105)$
Pain at

baseline

$(n=45)$
No pain at baseline

$(n=60)$

Time to BPI-SF deterioration, months

Worst pain/time to pain progression

\section{6 [4.7-9]}

$7.9[6.4-9.4]$

Least pain

Average pain

Overall pain interference

\begin{tabular}{ll} 
Median & $5.6[4.7-9]$ \\
Mean & $7.9[6.4-9.4]$ \\
\hline Median & $7.1[6.2-\mathrm{NR}]$ \\
\hline Mean & $10.7[8.5-12.9]$
\end{tabular}

Median

6.1 [5.5-NR]

Mean

$9.4[7.8-11]$

Median

$6.2[4.7-N R]$

9 [7.3-10.6]

Median

Mean

Clinically meaningful improvement of BPI-SF Worst pain during treatment, no. of patients (\%)

Pain response, no. of patients (\%)

Complete

Partial

Indeterminate

Progressive pain

Not evaluable

Time to FACT-P deterioration, months

Total

Physical well-being

Emotional well-being

Pain

$\begin{array}{ll}\text { Median } & 5.7[3.3-N R] \\ \text { Mean } & 7.8[6.2-9.3]\end{array}$

10.4 [8.2-12.5]

52 (49.5)

33 (31.4)

28 (26.7)

35 (33.3)

$6(5.7)$

$3(2.8)$

$7.8[6.2-9.3]$

9.8 [7-NR]

11.1 [8.9-13.2]

Mean

Median

Mean

NR [7.2-NR]

12.4 [10.4-14.4]

Median

13.2 [11.2-NR]

Mean

$13.2[11.1-15.3]$

Median

NR [NR-NR]

Mean

13.6 [12.1-15.2]

Median

NR [12.7-NR]

13.9 [12-15.9]

10.7 [9-NR]

Median

Mean

Clinically meaningful improvement of Total FACT-P during treatment, no. of patients (\%)

9.6 [7.9-11.3]

$33(31.4)$

$\begin{array}{ll}11.1[7.6-\mathrm{NR}] & 4.1[3.6-5.7] \\ 11.2[8.5-13.8] & 6.1[4.6-7.7]\end{array}$

14.1 [6.9-NR]

11.5 [8.3-14.7]

6.5 [5.8-NR]

$9.6[7.3-11.9]$

$12.6[6.2-\mathrm{NR}] \quad 5.5[4.1-6.8]$

$11.5[8.8-14.2]$

$8[6.1-9.8]$

NR [10-NR] $\quad 5.7[4.1-7.2]$

11.9 [9.1-14.6]

$7.7[5.8-9.6]$

$10.6[7.2-N R] \quad 6.7[5.7-N R]$

$9.9[7.1-12.8] \quad 9.8[7.5-12.1]$

35 (77.7)

17 (28.3)

$<0.0001$

0.004

0.03

0.0001

ns

ns

$3(6.7)$

7 (11.7)

$24(40.0)$

$3(5.0)$

2 (1.7)

5.5 [3.1-NR]

7 [5.4-8.6]

8.4 [6.4-10.5]

ns

9.8 [7-NR]

9.9 [7.5-12.3]

12.4 [9.6-15.2]

ns

NR [NR-NR]

12.8 [10.7-14.9]

10.2 [7-13.5]

ns

13.2 [10.4-NR]

12.3 [10-14.6]

14.6 [12.3-17]

NR [12.6-NR]

14.4 [12-16.8]

NR [NR-NR]

13.1 [11.2-15]

ns

NR [NR-NR]

12.7 [7.6-NR]

$12.4[9.2-15.6]$

12.6 [12.6-NR]

11 [8.9-13.1]

9 [5.8-NR]

8.3 [6.9-9.7]

17 (37.7)

16 (26.7)

ns

Clinically meaningful improvement of Total FACT-P was defined as a minimal change of 10 points from baseline for the Total FACT-P score, 3 points from baseline for the subscales and 2 points from baseline for pain. The Clinically Meaningful improvement of BPI-SF score (CMC-BPI) was defined as a change of score of at least $30 \%$ from baseline score, with a minimum of 2 points.

BPI-SF Brief Pain Inventory-Short Form, FACT-P Functional Assessment of Cancer Therapy-Prostate, NR not reached, ns not significant.

${ }^{a}$ Pain at baseline vs no-pain at baseline. 
A

Time to pain progression (Worst pain)

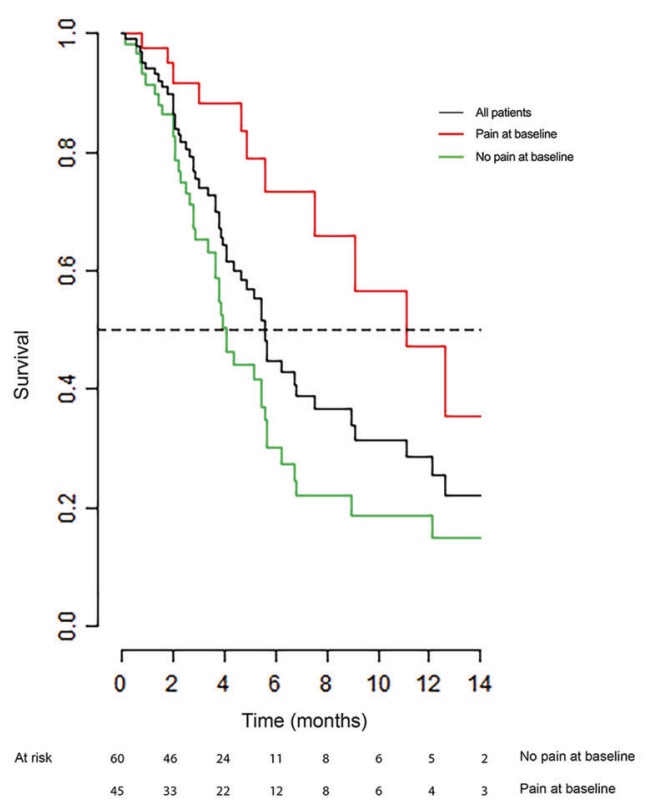

B

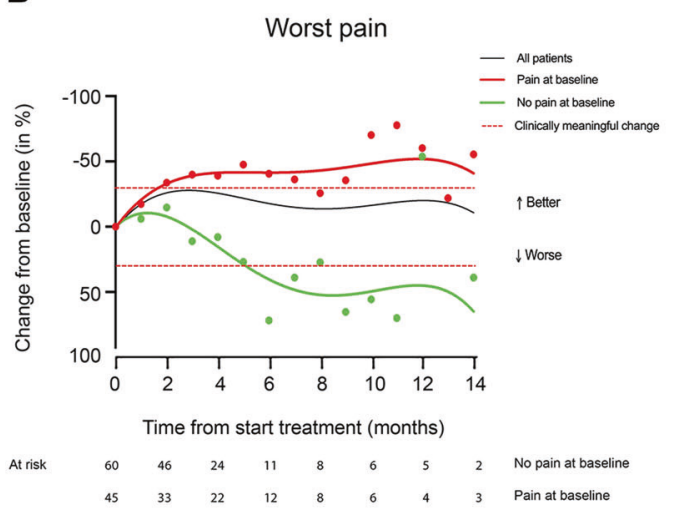

C

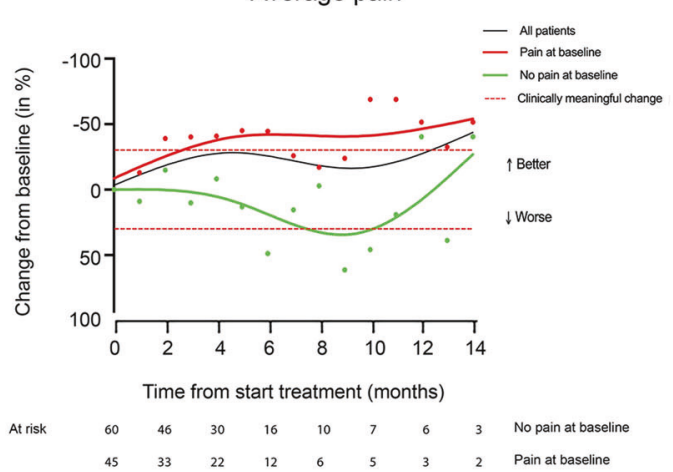

Fig. 1 Brief Pain Inventory (BPI). A Kaplan-Meier estimates of time to clinically meaningful Brief Pain Inventory Short Form (BPI-SF) Worst pain subscale score deterioration for the evaluable sample (black line), patients with pain at baseline (red line), and patients without pain at baseline (green line). The horizontal dotted line represents 50\% events. B Change in BPI-SF - Worst pain. C Average pain subscale scores over time in the evaluable sample (black line), patients with pain at baseline (red line), and patients without pain at baseline (green line). Data points show average scores at time points, while the lines are made to fit the trend of change of score in time. The horizontal dotted lines represent the threshold for clinically meaningful change from baseline.

With an estimated PROM response rate of $40 \%$, we aimed to include 300 patients.

Software. TENALEA, an online service, was used to collect data. IBM SPSS statistics for iOS, version 25 (IBM Corp. Released 2017, Version 25.0. Armonk, NY: IBM Corp.) and Statistical Analysis System (SAS) statistical software were used for statistical analysis and for constructing graphs. Additional graphs and analyses were made and performed using GraphPad Prism for iOS version 8.00, GraphPad Software, La Jolla, CA, USA, www.graphpad.com.

\section{RESULTS}

\section{Baseline characteristics and survival}

Between April 2015 and March 2018, 305 mCRPC patients from 20 Dutch hospitals scheduled for Ra-223 treatment were included. Five patients were excluded because written approval to use identifiers (name, address, residence) could not be retrieved or was not stored according to guidelines (Supplementary Fig. 1). This registry included 300 patients (registry sample), of whom 121 (40\%) completed the baseline questionnaires, and 105 (35\%) completed baseline and at least one follow-up BPI-SF and FACT-P questionnaire and were therefore evaluable for the individual questionnaires (evaluable sample). In all, 103 patients were evaluable for pain response analysis, because 2 patients provided insufficient data on analgesics use.

The registry sample and the evaluable sample were comparable on most baseline characteristics, survival characteristics, and treatment outcomes (Table 1 and Supplementary Table 4). However, patients in the evaluable sample significantly used calcium/ vitamin D supplementation more often, and bisphosphonates less often than patients in the registry sample. Moreover, evaluable patients less often received EBRT in the 12 weeks prior to Ra-223. Although there was no significant difference in PFS, OS was significantly shorter in the registry sample than in the evaluable sample (15.2 and 19.6 months, respectively, $p=0.04$ ).

Of the 105 evaluable patients, the majority received Ra-223 as a third or higher line MCRPC treatment and previously received docetaxel and abiraterone or enzalutamide (Table 1). Forty-five patients had PAB and 60 had no-PAB (Supplementary Fig. 1 and Table 1). The baseline characteristics of the two groups were comparable, however, more PAB patients used opioids $(51.2 \%$ and $16.7 \%$, respectively, $p<0,001$ ). After a median follow-up of the evaluable sample of 13.2 months, PAB patients had a significantly shorter OS than no-PAB patients (13.5 and 20.3 months, respectively, $p=0.05$ ) (Supplementary Table 4 and Supplementary Fig. 2).

Pain and health-related quality of life

Questionnaire completion rates per time point are listed in Supplementary Table 5.

BPI-SF. BPI-SF baseline values are reported in Supplementary Table 6. PAB patients scored significantly higher on all baseline BPI-SF subscales compared to no-PAB patients $(p<0.001)$. The percentage of patients experiencing a complete pain response for the duration of Ra-233 treatment was $31.4 \%$ (Table 2). Changes in time of the BPI-SF Worst pain and Average pain subscales are displayed in Fig. 1A, B, respectively, and the other BPI-SF subscales 
A

Time to Total FACT-P deterioration

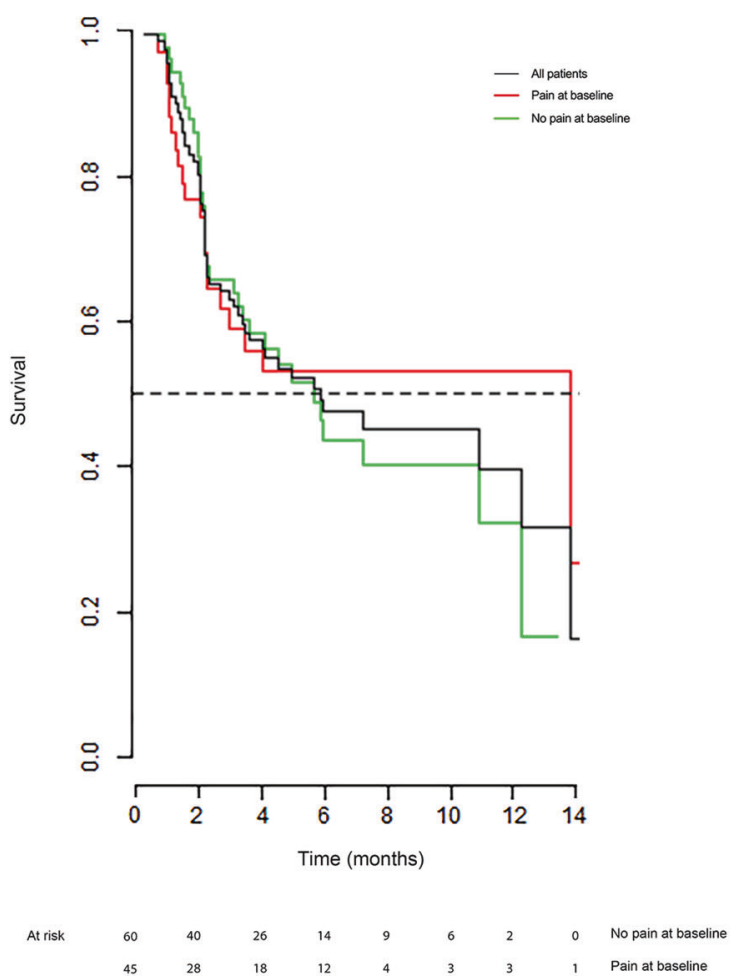

B

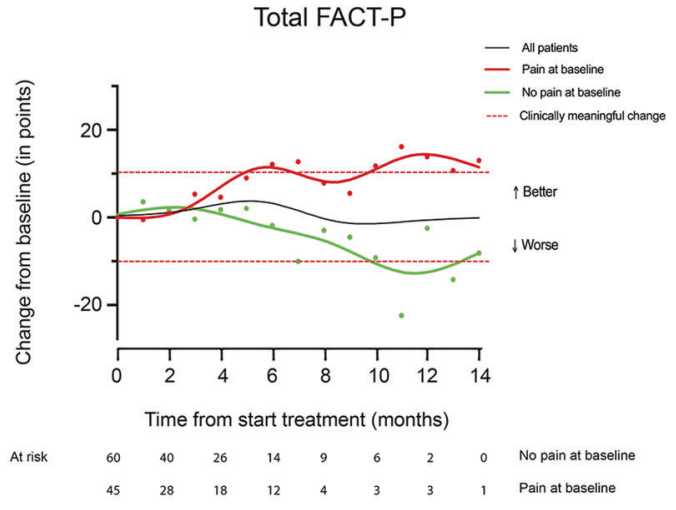

C

Prostate Cancer Subscale

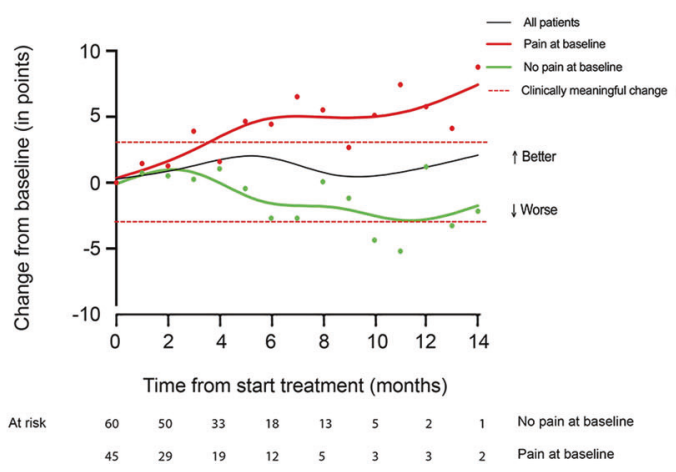

Fig. 2 Functional Assessment of Cancer Therapy-Prostate (FACT-P). A Kaplan-Meier estimates of time to clinically meaningful Total Functional Assessment of Cancer Therapy-Prostate (FACT-P) score deterioration for the evaluable sample (black line), patients with pain at baseline (red line), and patients without pain at baseline (green line). The horizontal dotted line represents 50\% events. B Change in Total FACT-P. C Prostate cancer subscale scores in time for the evaluable sample (black line), patients with pain at baseline (red line), and patients without pain at baseline (green line). Data points show average score at time points, while the lines are made to fit the trend of change of score in time. The horizontal dotted lines represent the threshold for clinically meaningful change from baseline.

in Supplementary Fig. 3. During treatment, $49.5 \%$ of the evaluable sample had a clinically meaningful improvement of the BPI-SF Worst pain subscale (Table 2 and Fig. 1B). Median and mean times to deterioration of the BPI-SF subscales are reported in Table 2 and Fig. 1A. PAB patients had a significantly longer median time to deterioration of the BPI-SF subscale Average pain than no-PAB patients. (Table 2 and Supplementary Fig. 4). PAB patients also had a significantly longer TPP than no-PAB patients (Table 2 and Fig. 1A).

FACT-P. FACT-P baseline values are reported in Supplementary Table 6. PAB patients had significantly lower baseline Total FACT-P scores than no-PAB patients $(95.2$ and 107.6 , respectively, $p<$ 0.001 ), suggesting a worse HRQoL.

During treatment, $31.4 \%$ of the evaluable sample had a clinically meaningful improvement of Total FACT-P, with no significant difference between PAB and no-PAB patients (Table 2 and Fig. 2B). Changes in time of the FACT-P subscales are displayed in Supplementary Fig. 5. Median and mean TTFD and other deteriorations of FACT-P subscales are reported in Table 2. There were no significant differences in deterioration times of Total FACT-P or the other FACT-P subscales between PAB and no-PAB patients. (Table 2, Fig. 2A, and Supplementary Fig. 6).

Analgesics use and integration of PROMs results. Use of analgesics in the evaluable sample decreased during Ra-223 treatment and remained low during follow-up (Fig. $3 \mathrm{~A}$ and Supplementary Fig. 7). The score of the BPI-SF subscale Worst pain did not show a clinically meaningful change during Ra-223 treatment and in follow-up. Ninety-five patients had sufficient data to be categorized for best pain response and Total FACT-P response. Fifty-five (57.9\%) had an IOCR, of whom $27(49.1 \%)$ were PAB and 28 (50.9\%) were no-PAB patients (Fig. 3B).

\section{DISCUSSION}

In the ALSYMPCA, pain was evaluated using the non-pain-specific questionnaires FACT-P and EQ-5D [5]. Evaluation of opioids use was limited to baseline opioid use and 3 monthly assessments of opioid use in patients without baseline use. A non-significant reduction in pain was found between Ra-223- and placebo-treated patients at 16 and 24 weeks of treatment $[4,5]$. The percentages of patients experiencing a clinically meaningful improvement of Total FACT-P in our cohort was comparable to ALSYMPCA $(31.4 \%$ and $24.6 \%$, respectively) [5]. However, there are critical differences between the ALSYMPCA population and the population in this cohort. The ALSYMPCA was conducted in a time when docetaxel was the only treatment option for MCRPC patients. Consequently, in ALSYMPCA, patients received Ra-223 after docetaxel or as a first-line $\mathrm{mCRPC}$ treatment. Contemporary $\mathrm{mCRPC}$ patients have multiple treatment options. In this study, more than half of the patients received at least 2 treatments prior to Ra-223 treatment. It can be assumed that the extensively pretreated patients in this study are prone to poorer performance, while strict patient selection might compensate for that. Moreover, in ALSYMPCA patients were symptomatic, while in this study the majority of patients had no-PAB. Unfortunately, baseline Total FACT-P scores of patients included in ALSYMPCA have not been made available 
A

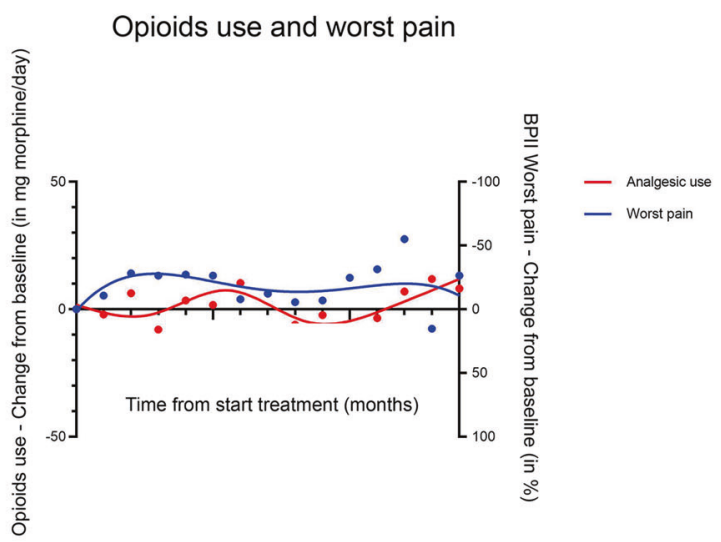

B

Best Pain and quality of life response

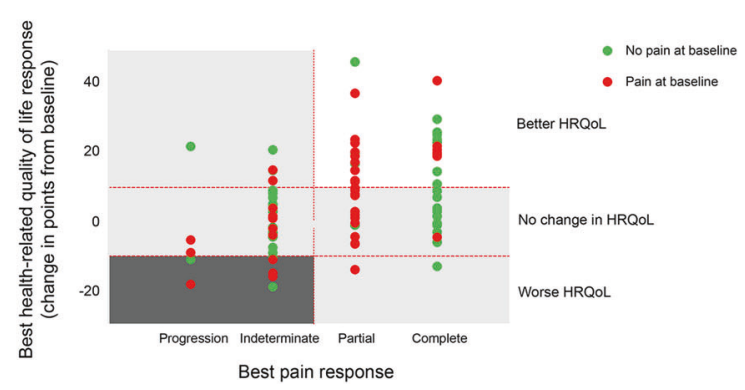

Fig. 3 Integrated pain and health-related quality of life response. A Percentage change in Brief Pain Inventory Short Form (BPI-SF) Worst pain subscale scores from baseline in time (blue line) and change in average analgesics use from baseline in $\mathrm{mg}$ morphine equivalents per day (red line). B Patients were categorized for their best pain response (Worst pain subscale) integrated with opioid drugs use according to IBMCWP recommendations (horizontal axis: progression, indeterminate, partial, and complete response) and for their best health-related quality of life response (vertical axis: Total FACT-P clinically meaningful better or worse or not meeting these criteria and therefore considered as no change). The red, horizontal dotted lines represent the threshold for clinically meaningful Total FACT-P change (10 points), while the vertical dotted line separates progression and indeterminate pain responses from partial and complete pain responses. Red dots represent pain at baseline patients and green dots no-pain at baseline patients.

$[5,16]$. In line with our results, three small retrospective studies, using various measurements, suggested that approximately half of the patients experience reduced pain during Ra-223 treatment [17-19]. One prospective study, using the cancer-specific EORTCQLQ-C30 measurement, showed no HRQoL deterioration during Ra-223 treatment [20].

In this study, outcomes of the different PROMs were integrated into an IOCR, which was established in $58 \%$ of patients. Cancerrelated pain and HRQoL are not mutually exclusive, as was reported previously [21, 22]. However, some patients had more pain but a better HRQoL, while others experienced less pain and a worse HRQoL. In part, this can be explained by inclusion of the best pain response and best HRQoL change for establishing the IOCR. Moreover, HRQoL can also be affected by other domains than pain, including fatigue, psychological distress, financial problems, or social problems [23]. Another possible explanation is that this is caused by response shift, where patients accommodate to their pain by cognitive reframing and reprioritizing of previously held values, internal standards, and expectations to help cope with high levels of pain [24].
The strength of this study lies in the inclusion of a contemporary real-world population, pretreated with multiple $\mathrm{mCRPC}$ treatment options. Moreover, both symptomatic and asymptomatic patients were included, as this inclusion criterion of the ALSYMPCA is generally not considered in daily practice. This makes the results of this study directly applicable to current prostate cancer patients' treatment. There is a growing interest in real-life data, however, PROMs are rarely reported. In line with the increased interest in PROMs outcomes from randomized trials, we would argue in favor of including these outcomes in real-life cohorts.

Limitations of this study include its non-randomized nature and the likelihood of survival and selection bias. Another limitation is the lower than expected questionnaire completion rates. The percentage of patients evaluable was within the previously reported $10-70 \%$ range of response rates in studies on selfreported outcome measures in real-life populations [25-27], but lower than the $40 \%$ we assumed for the power calculation. It was previously reported that a higher frailty score was a strong predictor for non-completion [28]. The older age and more advanced disease, and with that a presumably higher frailty score of patients in our cohort compared with similar studies in patients with other cancers, might explain the low completion rates. Despite the above, the evaluable sample seemed to be representative for the registry sample since there were no major differences in baseline characteristics.

In conclusion, our study shows that a significant proportion of Ra-223-treated symptomatic and asymptomatic, extensively pretreated $\mathrm{mCRPC}$ patients experience an improved HRQoL and pain response. These results suggest that the majority of contemporary mCRPC patients derive clinical benefit from Ra-223 treatment.

\section{DATA AVAILABILITY}

The data underlying this article will be shared on reasonable request to the corresponding author.

\section{REFERENCES}

1. Ferlay J, Colombet $M$, Soerjomataram I, Mathers C, Parkin DM, Piñeros $M$, et al. Estimating the global cancer incidence and mortality in 2018: GLOBOCAN sources and methods. Vol 144, Int J Cancer. 2019;144:1941-53. https://onlinelibrary. wiley.com/doi/full/10.1002/ijc.31937.

2. Sathianathen NJ, Koschel S, Thangasamy IA, Teh J, Alghazo O, Butcher G, et al. Indirect comparisons of efficacy between combination approaches in metastatic hormone-sensitive prostate cancer: a systematic review and network metaanalysis. Eur Urol. 2019;77:365-72. https://www.sciencedirect.com/science/ article/pii/S0302283819306931?via\%3Dihub.

3. Saad F, Ivanescu C, Phung D, Loriot Y, Abhyankar S, Beer TM, et al. Skeletal-related events significantly impact health-related quality of life in metastatic castrationresistant prostate cancer: data from PREVAIL and AFFIRM trials. Prostate Cancer Prostatic Dis. 2017;20:110-6. www.nature.com/pcan.

4. Parker C, Nilsson S, Heinrich D, Helle SI, O'Sullivan JM, Fosså SD, et al. Alpha emitter Radium-223 and survival in metastatic prostate cancer. N Engl J Med. 2013;369:213-23. http://www.nejm.org/doi/abs/10.1056/NEJMoa1213755.

5. Nilsson S, Cislo P, Sartor O, Vogelzang NJ, Coleman RE, O'Sullivan JM, et al. Patient-reported quality-of-life analysis of radium-223 dichloride from the phase III ALSYMPCA study. Ann Oncol. 2016;27:868-74. https://academic.oup.com/ annonc/article-lookup/doi/10.1093/annonc/mdw065.

6. Heidenreich A, Gillessen S, Heinrich D, Keizman D, O'Sullivan JM, Carles J, et al. Radium-223 in asymptomatic patients with castration-resistant prostate cancer and bone metastases treated in an international early access program. BMC Cancer. 2019;19:12. https://bmccancer.biomedcentral.com/articles/10.1186/ s12885-018-5203-y.

7. Teo MY, Rathkopf DE, Kantoff P. Treatment of advanced prostate cancer. Annu Rev Med. 2019;70:479-99. https://www.ncbi.nlm.nih.gov/pmc/articles/ PMC6441973/?report=abstract.

8. Badrising SK, Louhanepessy RD, vd Noort V, Coenen JL, Hamberg P, Beeker A, et al. A prospective observational registry evaluating clinical outcomes of Radium-223 treatment in a non-study population. Int J Cancer. 2020;147:1143-51. https://onlinelibrary.wiley.com/doi/abs/10.1002/ijc.32851. 
9. Fujimura T, Takahashi S, Kume H, Takeuchi T, Kitamura T, Homma Y. Cancerrelated pain and quality of life in prostate cancer patients: assessment using the Functional Assessment of Prostate Cancer Therapy. Int J Urol. 2009;16:522-5. http://doi.wiley.com/10.1111/j.1442-2042.2009.02291.x.

10. Cleeland CS, Ryan KM. Pain assessment: global use of the Brief Pain Inventory. Ann Acad Med Singap. 1994;23:129-38. http://www.ncbi.nlm.nih.gov/pubmed/ 8080219.

11. Atkinson TM, Mendoza TR, Sit L, Passik S, Scher HI, Cleeland C, et al. The Brief Pain Inventory and its "pain at its worst in the last $24 \mathrm{~h}$ " item: clinical trial endpoint considerations. Pain Med. 2010;11:337-46. http://www.ncbi.nlm.nih.gov/ pubmed/20030743.

12. Esper $P, M o ~ F$, Chodak G, Sinner M, Cella D, Pienta KJ. Measuring quality of life in men with prostate cancer using the Functional Assessment of Cancer Therapy-Prostate instrument. Urology. 1997;50:920-8. http://www.ncbi.nlm.nih.gov/pubmed/9426724.

13. Dworkin RH, Turk DC, Wyrwich KW, Beaton D, Cleeland CS, Farrar JT, et al Interpreting the clinical importance of treatment outcomes in chronic pain clinical trials: IMMPACT recommendations. J Pain. 2008;9:105-21. http://www. ncbi.nlm.nih.gov/pubmed/18055266.

14. Cella D, Nichol MB, Eton D, Nelson JB, Mulani P. Estimating clinically meaningful changes for the Functional Assessment of Cancer Therapy-Prostate: results from a clinical trial of patients with metastatic hormone-refractory prostate cancer. Value Heal. 2009;12:124-9. https://www.valueinhealthjournal.com/article/S1098-3015 (10)60683-3/pdf?_returnURL=https\%3A\%2F\%2Flinkinghub.elsevier.com\% 2Fretrieve\%2Fpii\%2FS1098301510606833\%3Fshowall\%3Dtrue.

15. Chow E, Hoskin P, Mitera G, Zeng L, Lutz S, Roos D, et al. Update of the international consensus on palliative radiotherapy endpoints for future clinical trials in bone metastases. Int J Radiat Oncol. 2012;82:1730-7. http://www.ncbi.nIm.nih. gov/pubmed/21489705.

16. Parker C, Finkelstein SE, Michalski JM, O'Sullivan JM, Bruland $\varnothing$, Vogelzang NJ, et al. Efficacy and safety of Radium-223 dichloride in symptomatic castrationresistant prostate cancer patients with or without baseline opioid use from the phase 3 ALSYMPCA trial. Eur Urol. 2016;70:875-83. http://www.ncbi.nlm.nih.gov/ pubmed/27344296.

17. De Luca R, Costa RP, Tripoli V, Murabito A, Cicero G. The clinical efficacy of Radium-223 for bone metastasis in patients with castration-resistant prostate cancer: an Italian clinical experience. Oncology. 2018;94:161-6. https://pubmed. ncbi.nlm.nih.gov/29241166/.

18. De Vincentis G, Frantellizzi V, Follacchio GA, Farcomeni A, Pani A, Samaritani R, et al. No evidence of association between psychological distress and pain relief in patients with bone metastases from castration-resistant prostate cancer treated with 223Radium. Eur J Cancer Care (Engl). 2019;28:e13112. https://onlinelibrary. wiley.com/doi/abs/10.1111/ecc.13112.

19. Parimi S, Bondy S, Tsang E, McKenzie MR, Bachand F, Aparicio M, et al. Pain response in a population-based study of radium-223 (Ra223) for metastatic castration-resistant prostate cancer. Can Urol Assoc J 2019;13:E311-6.

20. Sraieb M, Hirmas N, Conrad R, Marinova M, Essler M, Herrmann K, et al. Assessing the quality of life of patients with metastatic castration-resistant prostate cancer with bone metastases receiving [223Ra]RaCl2 therapy. Medicine (Baltim). 2020;99:e22287. https://www.ncbi.nlm.nih.gov/pmc/articles/PMC7505355/.

21. Ji A, Shi R, Han J, Yu C. Focus on cancer pain at home: a prospective study of quality of life and its influencing factors on patients. J Cancer Ther. 2019;10:815-28. http:// www.scirp.org/journal/doi.aspx?DOI=10.4236/jct.2019.1010069.

22. Hjermstad MJ, Kaasa S, Caraceni A, Loge JH, Pedersen T, Haugen DF, et al Characteristics of breakthrough cancer pain and its influence on quality of life in an international cohort of patients with cancer. BMJ Support Palliat Care 2016;6:344-52. http://www.ncbi.nlm.nih.gov/pubmed/27342412.

23. Colloca $G$, Colloca $P$. The effects of social support on health-related quality of life of patients with metastatic prostate cancer. J Cancer Educ. 2016;31:244-52.

24. Ilie G, Bradfield J, Moodie L, Lawen T, llie A, Lawen Z, et al. The role of responseshift in studies assessing quality of life outcomes among cancer patients: a systematic review. Front Oncol. 2019;9:783. https://www.frontiersin.org/article/ 10.3389/fonc.2019.00783/full.

25. Baruch $Y$, Holtom BC. Survey response rate levels and trends in organizational research. Hum Relat. 2008;61:1139-60. http://journals.sagepub.com/doi/10.1177/ 0018726708094863.

26. Husson $\mathrm{O}$, de Rooij BH, Kieffer J, Oerlemans S, Mols F, Aaronson NK, et al. The EORTC QLQ-C30 summary score as prognostic factor for survival of patients with cancer in the "Real-World": results from the population-based PROFILES Registry. Oncologist. 2020;25:e722-32. http://www.ncbi.nlm.nih.gov/pubmed/31672773.
27. Sinclair M, Otoole J, Malawaraarachchi M, Leder K. Comparison of response rates and cost-effectiveness for a community-based survey: postal, Internet and telephone modes with generic or personalised recruitment approaches. BMC Med Res Methodol. 2012;12:132.

28. Hohwü L, Lyshol H, Gissler M, Jonsson SH, Petzold M, Obel C. Web-based versus traditional paper questionnaires: a mixed-mode survey with a nordic perspective. J Med Internet Res. 2013;15:e173.

\section{ACKNOWLEDGEMENTS}

In addition to the authors, the following investigators contributed to this study (in alphabetical order): F. vd Berkmortel, H.J. Bloemendal, W. vd Deure-Gielisse, N. Hagen, H.H. Helgason, C.J. Hoekstra, D. Houtsma, B. de Keizer, J.M.H. de Klerk, K.P. Koopmans, B. Kuenen, M. Los, L. Prompers, T.A. Roeleveld, W. Schreurs, T. Smilde, W. Vogel, T. van Voorthuizen, and D.N.J Wyndaele. We also thank the members of the Dutch UroOncology Study group (DUOS15102).

\section{FUNDING}

This work was supported by a grant from Bayer BV, Mijdrecht, The Netherlands.

\section{Declarations (Ethics)}

\section{COMPETING INTERESTS}

A.M.B. participated in Advisory Boards of Janssen Pharma, Bayer, Sanofi and Astellas, received speaking fees from Jansen Pharma, Bayer and Astellas, and received research grants from Sanofi and Astellas. J.M.F. participated in advisory boards of Janssen Pharma, Merck, and Pfizer. J.B.H. has provided consultation, attended advisory boards, and/or provided lectures for AIMM, Amgen, BioNTech, BMS, GSK, Ipsen, Merck Serono, Molecular Partners, MSD, Novartis, Pfizer, Roche/Genentech, Sanofi, Seattle Genetics, and Third Rock ventures. He is on the scientific advisory boards of IMM, BioNTech US, Gadeta, Immunocore, T-Knife and Neogene Therapeutics. He received grant support from Amgen, BioNTech US, BMS, MSD, and Novartis. He also has stock options in Neogene Therapeutics. All the remaining authors declare no competing interests.

\section{ADDITIONAL INFORMATION}

Supplementary information The online version contains supplementary material available at https://doi.org/10.1038/s41391-021-00412-6.

Correspondence and requests for materials should be addressed to W.Z. or A.M.B.

Reprints and permission information is available at http://www.nature.com/ reprints

Publisher's note Springer Nature remains neutral with regard to jurisdictional claims in published maps and institutional affiliations.

\footnotetext{
Open Access This article is licensed under a Creative Commons Attribution 4.0 International License, which permits use, sharing, daptation, distribution and reproduction in any medium or format, as long as you give appropriate credit to the original author(s) and the source, provide a link to the Creative Commons license, and indicate if changes were made. The images or other third party material in this article are included in the article's Creative Commons license, unless indicated otherwise in a credit line to the material. If material is not included in the article's Creative Commons license and your intended use is not permitted by statutory regulation or exceeds the permitted use, you will need to obtain permission directly from the copyright holder. To view a copy of this license, visit http://creativecommons. org/licenses/by/4.0/.
}

(c) The Author(s) 2021 